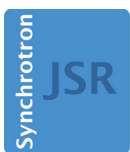

JOURNAL OF SYNCHROTRON RADIATION

ISSN 1600-5775

Received 30 July 2019

Accepted 11 November 2019

Edited by I. Schlichting, Max Planck Institute for Medical Research, Germany

Keywords: Elettra synchrotron; Xpress beamline; single-crystal XRD; high pressure.

Supporting information: this article has supporting information at journals.iucr.org/s
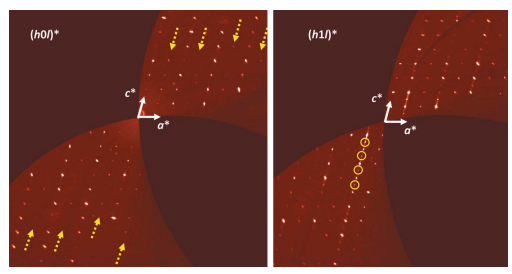

C 2020 International Union of Crystallography

\section{Single-crystal diffraction at the high-pressure Indo-Italian beamline Xpress at Elettra, Trieste}

\author{
Paolo Lotti, ${ }^{\mathrm{a}, \mathrm{b}}$ Sula Milani, ${ }^{\mathrm{a}}$ Marco Merlini, ${ }^{\mathrm{a} *}$ Boby Joseph, \\ Frederico Alabarse $^{\mathbf{b}}$ and Andrea Lausi ${ }^{\mathrm{b}}$
}

${ }^{a}$ Dipartimento di Scienze della Terra, Università degli Studi di Milano, Via Botticelli 23, 20133 Milano, Italy,

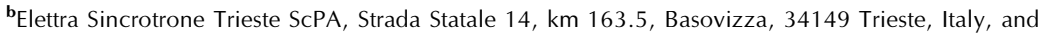
${ }^{{ }^{c}}$ Research Group IISc-ICTP, Strada Statale 14, km. 163.5, Basovizza, 34149 Trieste, Italy.

*Correspondence e-mail: marco.merlini@unimi.it

In this study the first in situ high-pressure single-crystal X-ray diffraction experiments at Xpress, the Indo-Italian beamline of the Elettra synchrotron, Trieste (Italy), are reported. A description of the beamline experimental setup and of the procedures for single-crystal centring, data collection and processing, using diamond anvil cells, are provided. High-pressure experiments on a synthetic crystal of clinoenstatite $\left(\mathrm{MgSiO}_{3}\right), \mathrm{CaCO}_{3}$ polymorphs and a natural sample of leucophoenicite $\left[\mathrm{Mn}_{7} \mathrm{Si}_{3} \mathrm{O}_{12}(\mathrm{OH})_{2}\right]$ validated the suitability of the beamline experimental setup to: (i) locate and characterize pressure-induced phase transitions; (ii) solve ab initio the crystal structure of high-pressure polymorphs; (iii) perform fine structural analyses at the atomic scale as a function of pressure; (iv) disclose complex symmetry and structural features undetected using conventional X-ray sources.

\section{Introduction}

High-pressure diffraction grew, during the last decades, from being a niche pioneering method to a widely used multidisciplinary technique. In Earth sciences, in situ high-pressure $(\mathrm{H} P)$ diffraction experiments are needed to determine accurate elastic constants of minerals, by fitting the experimental volume-pressure $(V-P)$ data by equations of state (Duffy \& Wang, 1998; Angel, 2000), as well as to explore phase transitions, shedding light on the potential mineralogy of the Earth's interior (Boffa Ballaran et al., 2013; Dubrovinsky et al., 2010; Dera, 2010). In materials science, high-pressure diffraction allows to disclose the relationships between the crystal structure and the physical-chemical properties of crystalline compounds and induce phase transitions to polymorphs of industrial or technological interest (e.g. McMillan, 2002; Boldyreva, 2008). Most of the problems addressed by highpressure experiments require, to be properly understood, a structural knowledge at the atomic scale. Single-crystal diffraction at high pressure is one of the most relevant techniques for understanding the atomic-scale behavior of matter at non-ambient conditions. However, the limited size of the pressure chambers of diamond anvil cells (DACs) (e.g. Miletich et al., 2000), the absorption of primary and diffracted $\mathrm{X}$-ray radiation by the diamond anvils, and the shadowing of significant portions of the reciprocal lattice by the metallic components of the DAC may pose severe limitations on the results achievable by in situ high-pressure single-crystal X-ray diffraction experiments. Synchrotron radiation allows singlecrystal X-ray diffraction to be performed on very small samples, allowing these experiments in a wide range of pres- 
sure and temperature (T) (McMahon et al., 2013; Merlini \& Hanfland, 2013; Dera et al., 2013; Dubrovinsky et al., 2010), possibly even using several samples loaded in a single DAC (Merlini et al., 2015; Yuan \& Zhang, 2017). This may be crucial, for example, when only natural or synthetic samples of very small size $(\sim 5-20 \mu \mathrm{m})$ are available or when the solution of complex crystal structures requires the simultaneous collection of diffraction data from at least two crystals with different orientation within the same DAC. The requirements for successful single-crystal diffraction experiments concern the stability of the X-ray source, low divergence of the beam, as well as the mechanical stability of the goniometer system. We here report the first single-crystal measurements at the Xpress beamline of the Elettra synchrotron, the Italian national synchrotron facility. Three examples will illustrate the feasibility of single-crystal diffraction at the beamline, with data of a suitable quality for performing structure refinements, structure solution and equation-of-state determination. These examples concern the structure refinement of low- and highpressure polymorphs of clinoenstatite, the structure determination of the high-pressure polymorphs of $\mathrm{CaCO}_{3}$, and the equation of state of the mineral leucophenicite.

\section{Beamline description}

The high-pressure Xpress beamline endstation makes use of the X-ray beam produced by a multipole superconducting wiggler, operating with a magnetic field of $3.5 \mathrm{~T}$. Primary carbon filters act as high-bandpass filters, cutting the energy below $4 \mathrm{keV}$. A primary beam splitter allows $0.5 \mathrm{mrad}$ of radiation in the horizontal plane to intercept the monochromator, a liquid-nitrogen-cooled $\mathrm{Si}(111)$ single crystal. The fixed operating energy for the endstation is $25 \mathrm{keV}(\lambda \simeq$ $0.5 \AA$ ). The beam is focused on the sample by a toroidal Ptcoated mirror at $33 \mathrm{~m}$ from the source and $12 \mathrm{~m}$ from the sample. The final beam size on the sample is approximately $0.08 \mathrm{~mm} \times 0.08 \mathrm{~mm}$. This size can be reduced by adding sets of secondary slits and pinholes.

The endstation is equipped with a three-axis motorized stage mounted on an $\omega$-rotation goniometer. An additional translational motorized stage allows to center the goniometer vertical axis on the $\mathrm{X}$-ray primary beam.

The detector is a fast online MAR345 image-plate scanner, which can be positioned at variable sample-to-detector distances, in the range $150-500 \mathrm{~mm}$. The beamline is equipped with an on-line ruby fluorescence detector system for pressure measurement and an automatic pressure controller for membrane-type DACs (Alabarse et al., 2019).

\section{Sample alignment, single-crystal $X$-ray diffraction data collection and data processing}

The sample is aligned by direct beam absorption. Assuming a reference system with orthogonal axes, where the $x$-axis is parallel to the primary beam, $y$ is in the horizontal plane and $z$ in the vertical plane, motorized scans along the $y$ and $z$ directions, monitored by a photodiode, allows the centering of the gasket hole and, possibly, of the single crystal. Scans along the $y$-axis, performed at variable $\omega$ angles ( $\omega$ being the angle made by the goniometer with rotation axis parallel to $z$ ), allow to compute the displacement of the sample from the rotation axis along the $x$ direction and to correct accordingly.

Single-crystal diffraction step-scans are performed by $\omega$ step rotation, using the standard protocols for single-crystal data collections. The control software computes the goniometer motor acceleration time to reach a constant speed at the selected angle, where the fast shutter opens and the MAR image plate detector accumulates the X-ray signal during the selected $\omega$-step rotation angle. The fast shutter closes at the end of the $\omega$-step angle and the goniometer motor decelerates to zero speed. At this point, the MAR detector reads the image plate and data are stored on a server.

A scan speed for step-scan data between 0.25 and $1^{\circ} \mathrm{s}^{-1}$ assures the best conditions for shutter synchronization, motor movement and diffraction data collection. To avoid saturation, normally $\mathrm{Al}$ or Fe filters are inserted into the primary beam. For single crystals of $30 \mu \mathrm{m}$ thickness a reduction of the primary beam by a factor of 10 to 100 is needed, depending on the actual crystal size and scattering power of the sample. Diamond diffraction saturates the detector, but this is not a major problem with a MAR imaging plate detector. Depending on the total number of frames and detector resolution used, a data collection lasts from 0.5 to $2 \mathrm{~h}$. For example, a strategy adopting a $1^{\circ}$ step-scan, $4 \mathrm{~s}$ per degree accumulation time, $60^{\circ} \omega$-rotation and mar2300 resolution (full detector area, i.e. $345 \mathrm{~mm}$ in diameter, and $150 \mu \mathrm{m} \times 150 \mu \mathrm{m}$ pixel size) requires $85 \mathrm{~min}$. Using the mar1600 mode $(150 \mu \mathrm{m} \times 150 \mu \mathrm{m}$ pixel size and $240 \mathrm{~mm}$ diameter active area) the data collection lasts $45 \mathrm{~min}$.

Single-crystal data reduction is performed with the Crysalis software (Rigaku Oxford Diffraction, 2018).

\section{Single-crystal structure refinement of $P 2_{1} / C$ and C2/c clinoenstatite}

Clinoenstatite is the monoclinic polymorph of enstatite, $\mathrm{MgSiO}_{3}$, a mineral which represents one of the major constituents of planetary materials. The ambient-pressure $P 2_{1} / c$ structure transforms into a $C 2 / c$ structure above $7 \mathrm{GPa}$ at ambient temperature. The $\mathrm{H} P$ polymorph represents the thermodynamic stable structure of enstatite at mantle conditions. Despite the relevance of these phases, only few data on the elastic and structural behavior of $\mathrm{H} P$ clinoenstatite above $10 \mathrm{GPa}$ exist (Lazarz et al., 2019). We report here crystal structure refinements of $P 2_{1} / c$ and $C 2 / c$ clinoenstatite in the pressure interval 0-20 GPa. The results are compared with those of Lazarz et al. (2019).

A synthetic single crystal of clinoenstatite was synthesized using a multi-anvil apparatus at the Earth Sciences Department of the University of Milan at $\sim 6 \mathrm{GPa}$ and $1200^{\circ} \mathrm{C}$ for $410 \mathrm{~h}$. To synthesize the clinoenstatite a gel, prepared following the procedure by Hamilton \& Henderson (1968), was used as a starting material. A single crystal $(\sim 30 \mu \mathrm{m} \times$ $20 \mu \mathrm{m} \times 20 \mu \mathrm{m})$, together with ruby spheres, was loaded in the 
pressure chamber of an Almax membrane-type DAC. The DAC is supplied with Boheler-Almax-designed diamond anvils, with a culet size of $300 \mu \mathrm{m}$ in diameter and a $60^{\circ}$ opening window. The pressure-transmitting medium loaded in the DAC was $\mathrm{Ne}$ and the pressure was monitored by the ruby fluorescence method (Mao et al., 1986; Chervin et al., 2001), using the Betsa PRL system available at the beamline. The strategy used to collect X-ray single-crystal diffraction data was: $1^{\circ}$ per frame $\omega$-step scan, counting time of $4 \mathrm{~s}$ per frame, in the angular interval $+30 /-30^{\circ}$.

Single-crystal diffraction data of clinoenstatite up to $7 \mathrm{GPa}$ are indexed with a primitive monoclinic cell (Table 1 ) and the transition to a $C$-centered cell is observed above this pressure point. Unit-cell volume and structural data (polyhedral volumes) as a function of pressure are reported in Fig. 1. Overall, these data are in agreement with the recently reported single-crystal results (Lazarz et al., 2019) and indicate a different compressibility for the octahedral cations polyhedra as a function of pressure (Fig. 1; Table 1).
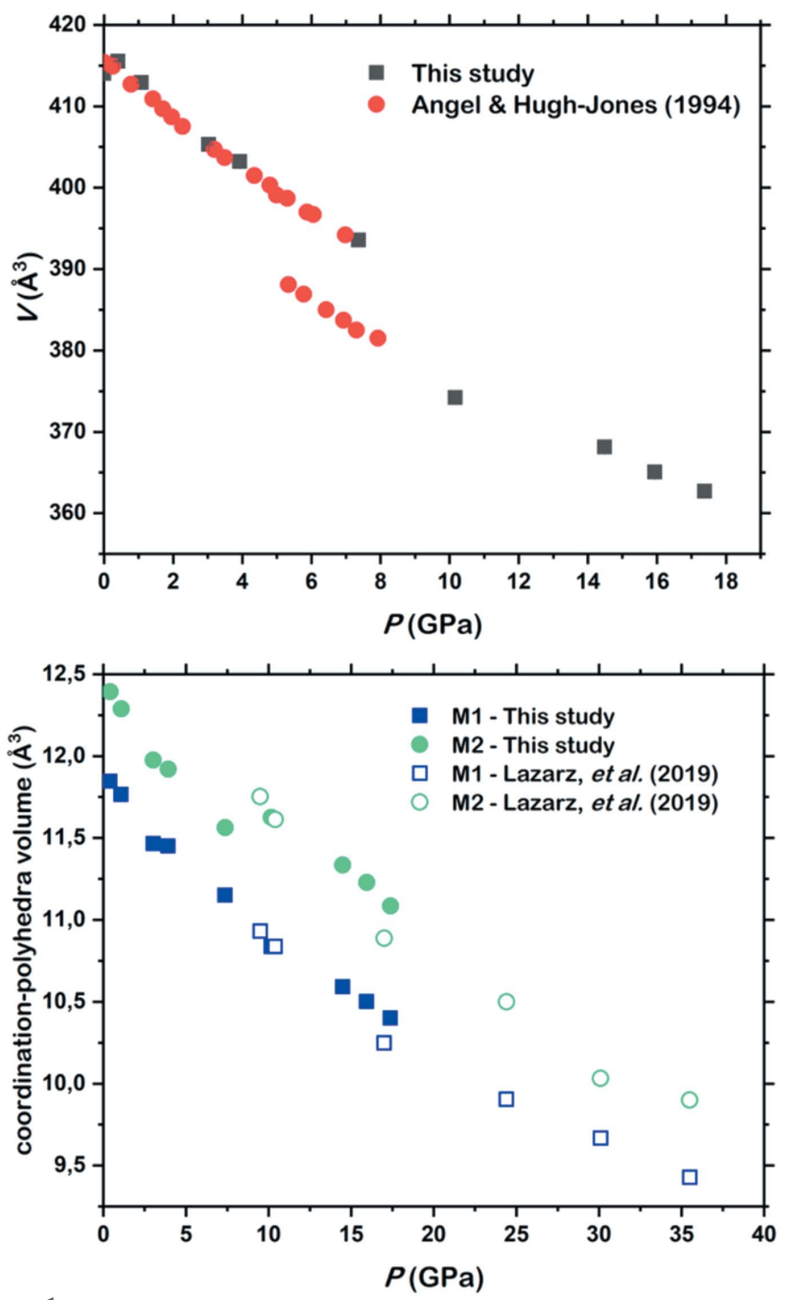

Figure 1

(Top) High-pressure evolution of the unit-cell volume of clinoenstatite, based on the results of this study and of Angel \& Hugh-Jones (1994). (Bottom) The volumes of the octahedral cations coordination-polyhedra as a function of pressure, based on the results of this study and of Lazarz et al. (2019).
Table 1

Lattice parameters of clinoenstatite at different pressures (pressure uncertainty: $\pm 0.1 \mathrm{GPa}$ ).

Standard deviations are given in parentheses.

\begin{tabular}{llllll}
\hline$P(\mathrm{GPa})$ & $a(\AA)$ & $b(\AA)$ & $c(\AA)$ & $\beta\left(^{\circ}\right)$ & $V\left(\AA^{3}\right)$ \\
\hline 0.41 & $9.3709(7)$ & $8.8117(6)$ & $5.1701(3)$ & $103.26(6)$ & $415.53(5)$ \\
1.08 & $9.352(2)$ & $8.794(1)$ & $5.1597(4)$ & $103.35(9)$ & $412.91(8)$ \\
3.03 & $9.313(2)$ & $8.730(2)$ & $5.1278(5)$ & $103.5(1)$ & $405.3(1)$ \\
3.93 & $9.299(2)$ & $8.710(2)$ & $5.1190(4)$ & $103.603(9)$ & $403.18(8)$ \\
7.37 & $9.256(2)$ & $8.618(2)$ & $5.0796(7)$ & $103.75(2)$ & $393.6(2)$ \\
10.17 & $9.147(1)$ & $8.554(2)$ & $4.8762(4)$ & $101.26(2)$ & $374.2(2)$ \\
14.49 & $9.082(9)$ & $8.503(1)$ & $4.8563(3)$ & $101.019(8)$ & $368.12(9)$ \\
15.95 & $9.064(2)$ & $8.462(2)$ & $4.8463(5)$ & $100.90(2)$ & $365.03(8)$ \\
17.38 & $9.039(1)$ & $8.447(2)$ & $4.8367(4)$ & $100.85(9)$ & $362.69(8)$ \\
\hline
\end{tabular}

\section{Single-crystal structural determination of $\mathrm{CaCO}_{3}$ high-pressure polymorphs}

$\mathrm{CaCO}_{3}$ high-pressure polymorphism has been studied since the pioneering high-pressure works of Bridgman (1939). The author discovered the transformation of calcite into highpressure phases different from aragonite on increasing pressure. In the 1960s, optical observation on calcite under high pressure induced by piston-type DAC revealed that applied pressures of less than $2 \mathrm{GPa}$ induced a second-order phase transition, without crystal disruption. Indeed, the optical Becke line crossed the entire crystal, which survived after transition (Van Valkenburg, 1965; Merrill \& Bassett, 1975). In the 1970s, Merrill and Bassett designed a miniaturized DAC which could be mounted on X-ray single-crystal diffractometers, to study and solve the high-pressure phase of calcite, $\mathrm{CaCO}_{3}$-II (Merrill \& Bassett, 1974; Bassett, 2009). $\mathrm{CaCO}_{3}$-II, stabilized above $1.5 \mathrm{GPa}$, is a distortion of the calcite structure. From a symmetry point of view, the space group of $\mathrm{CaCO}_{3}$-II $\left(P 2_{1} / c\right)$ is a subgroup of calcite space group $(R \overline{3} c)$. The three-fold axis is no longer present and the carbonate groups in the structure, still planar and parallel, are rotated by an angle compared with the calcite structure (Fig. 2). The Merrill-Bassett DAC readily became the most popular device for performing X-ray single-crystal diffraction at high pressure. Above $2.1 \mathrm{GPa} \mathrm{CaCO}_{3}$-II transforms to a higherpressure polymorph, $\mathrm{CaCO}_{3}$-III. The crystal structure determination of $\mathrm{CaCO}_{3}$-III represented an issue in highpressure crystallography for almost 40 years. Several models have been proposed (e.g. Davis, 1964; Liu \& Mernagh, 1990; Fiquet et al., 1994; Smyth \& Ahrens, 1997; Suito et al., 2001; Hagiya et al., 2005) but none was actually correct. The structure was solved more recently (Merlini et al., 2012) combining synchrotron radiation, large area detectors and the introduction of new algorithms for structure solution (Oszlányi \& Sütő, 2004; Palatinus \& Chapuis, 2007). Actually, as noted by Oganov et al. (2006), there are a number of $\mathrm{CaCO}_{3}$ highpressure structures which possess similar energy which can be stabilized experimentally. Two different structures based on the same topology have been observed above $2.1 \mathrm{GPa}$, both triclinic, $P \overline{1}$, called $\mathrm{CaCO}_{3}$-III and $\mathrm{CaCO}_{3}$-IIIb. Some experiments promote the formation of $\mathrm{CaCO}_{3}$-III, other experimental runs have $\mathrm{CaCO}_{3}$-IIIb and, finally, both polymorphs 
Table 2

Lattice parameters and space group for different polymorphs of calcite at different pressures.

Standard deviations are given in parentheses.

\begin{tabular}{|c|c|c|c|c|c|c|c|c|}
\hline$P(\mathrm{GPa})$ & $a(\AA)$ & $b(\AA)$ & $c(\AA)$ & $\alpha\left(^{\circ}\right)$ & $\beta\left(^{\circ}\right)$ & $\gamma\left({ }^{\circ}\right)$ & $V\left(\AA^{3}\right)$ & Space group \\
\hline $0.00001(1)$ & $4.9917(7)$ & 4.9917 (7) & $17.062(4)$ & 90 & 120 & 90 & $368.2(2)$ & $R \overline{3} c$ \\
\hline $1.77(2)$ & $6.276(2)$ & $4.993(2)$ & $7.907(4)$ & 90 & 107.25 & 90 & $236.7(2)$ & $P 2_{1} / c$ \\
\hline $2.69(2)$ & 6.295 & $7.536(3)$ & $12.512(5)$ & $93.36(3)$ & $98.92(2)$ & $106.74(2)$ & $558.1(4)$ & $P \overline{1}$ \\
\hline
\end{tabular}

a)

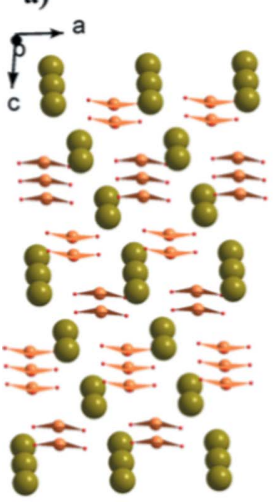

b)

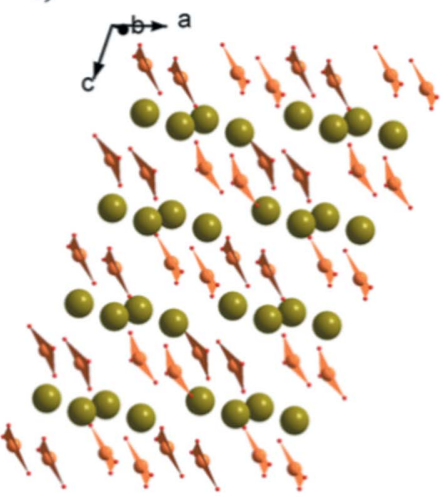

c)

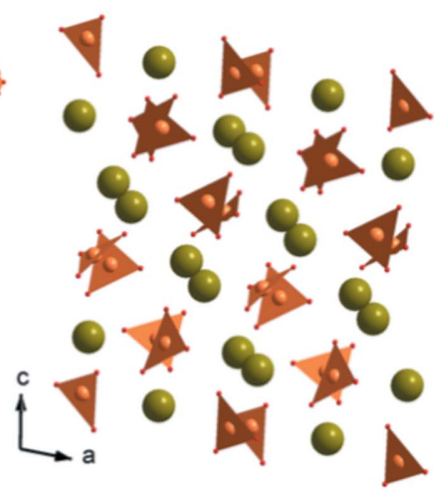

Figure 2

Structure of calcite and its high-pressure polymorphs. (a) $\mathrm{CaCO}_{3}$ stable up to $1.5 \mathrm{GPa}$ (space group $R \overline{3} c)$. (b) $\mathrm{CaCO}_{3}$-II $\left(P 2_{1} / c\right)$, stable up to $2.1 \mathrm{GPa}$, is a subgroup of calcite space group $(R \overline{3} c)$. (c) $\mathrm{CaCO}_{3}$-III $(P \overline{1})$ polymorph. Ca cations are represented by green spheres, while the $\mathrm{CO}_{3}$ planar groups are in orange.

have been observed simultaneously or transforming one into the other on pressure and/or temperature variation. Both $\mathrm{CaCO}_{3}$-III and $\mathrm{CaCO}_{3}$-IIIb structures can be considered as superstructures of a face-centred-cubic $\mathrm{Ca}$ atomic arrangement, with the $\mathrm{CO}_{3}$ groups occupying the interstices. The carbonate groups are non-parallel to each other and the different tilting creates possible different superstructures. $\mathrm{CaCO}_{3}$-III and $\mathrm{CaCO}_{3}$-IIIb, with 50 and 40 atoms, respectively, in the unit cell, are usually observed within the 2.1$15 \mathrm{GPa}$ interval and no other structures have been reported yet in this interval at room temperature. The transition from $\mathrm{CaCO}_{3}$-II to $\mathrm{CaCO}_{3}$-III is first-order in character, with a volume discontinuity. Usually, the transition induces the nucleation of several single-crystal domains, characterized by small size and different orientation.

We performed single-crystal X-ray diffraction data collections on calcite, $\mathrm{CaCO}_{3}$-II and $\mathrm{CaCO}_{3}$-III at the Xpress beamline as a useful test to validate the possibility of performing in situ single-crystal X-ray diffraction at high pressure after multiple phase transitions, aimed to a full structural determination of the investigated sample. The results are reported here.

A single crystal of $\mathrm{CaCO}_{3}, \sim 50 \mu \mathrm{m} \times 50 \mu \mathrm{m} \times 30 \mu \mathrm{m}$ size, was loaded into an Almax membrane-type DAC. The DAC is equipped with Boheler-Almax-designed diamond anvils, with a culet size of $600 \mu \mathrm{m}$ in diameter, and a stainless-steel gasket, pre-indented to $80 \mu \mathrm{m}$ in thickness and with a hole of $250 \mu \mathrm{m}$ in diameter obtained by spark-erosion. The adopted pressuretransmitting medium was silicone oil and pressure was moni- tored by the ruby fluorescence method (Mao et al., 1986; Chervin et al., 2001), using the Betsa PRL system available at the beamline.

X-ray single-crystal diffraction data were collected adopting the following strategy: a $1^{\circ}$ per frame $\omega$-step scan, with a counting time of $4 \mathrm{~s}$ per frame, in the angular interval $+30 /-30^{\circ}$. The primary X-ray beam $(80 \mu \mathrm{m}$ in diameter) was attenuated by a factor of 40 for the data collection on calcite and $\mathrm{CaCO}_{3}$-II and by a factor of 10 for the data collection of $\mathrm{CaCO}_{3}$-III.

The structure determinations and refinements of $\mathrm{CaCO}_{3}$ polymorphs were successful, using the charge flipping algorithm and difference-Fourier analysis for structure solution, implemented in the JANA2006 software package (Petřǐček et al., 2014). The results are summarized in Table 2 and in the cif files (see the supporting information).

\section{High-pressure elastic behavior, symmetry and structure determination of leucophoenicite by single-crystal diffraction}

Understanding and modeling the behavior of mantle minerals and rocks is achieved through different analytical and computational techniques. Among others, in situ X-ray diffraction studies at non-ambient conditions may provide an accurate determination of the thermodynamic elastic parameters and a description of the structural modifications accommodating the bulk compression or expansion. When available, the mineral phase of interest is directly investigated, but an additional understanding of the crystal chemical control on the compressional behavior can be derived by analyzing also chemically and/or structurally related compounds. For example, anhydrous, hydrous and superhydrous $\mathrm{B}$ phases, dense hydrous $\mathrm{Mg}$-silicates which may act as stable $\mathrm{H}_{2} \mathrm{O}$-storage phases at mantle conditions (e.g. Finger et al., 1989), are structurally related to the humite and leucophoenicite families of minerals (e.g. Makovicky, 1995). In particular, the hydrous double $(\mathrm{Mg})$-octahedral layer of phase-B is topologically identical to the double (Mn)-octahedral layer of leucophoenicite (Fig. 3), with the difference that a further anhydrous ( $\mathrm{Si}, \mathrm{Mg})$-octahedral layer is present in the former phase and absent in the latter. It follows that a 


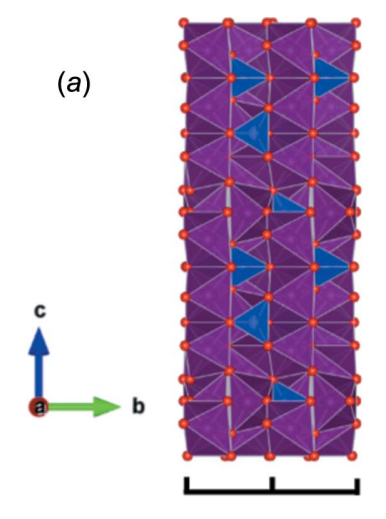

(c)
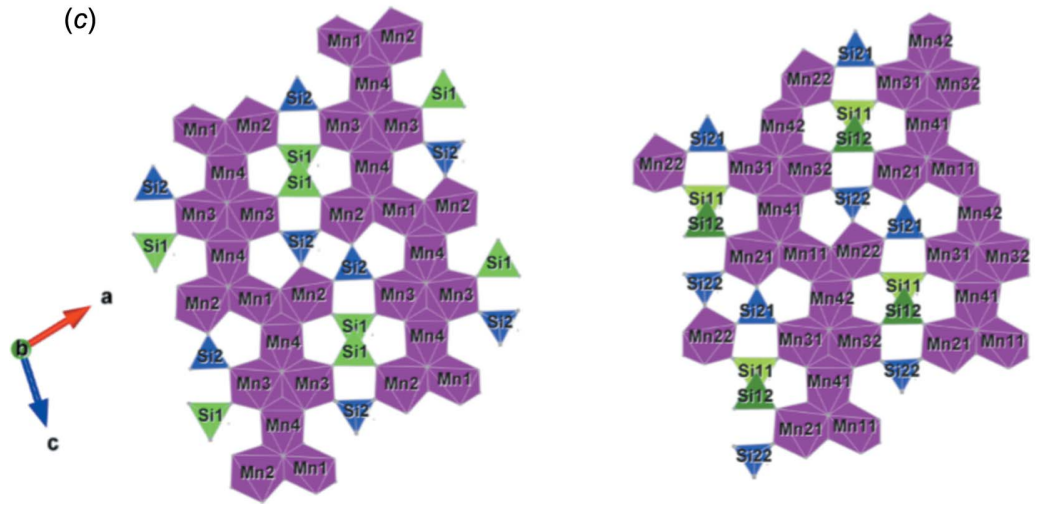

Figure 3

(a) The leucophoenicite structure viewed down [100]: the double octahedral layers stacking along the $b$ axis are shown. (b) A fragment of the leucophoenicite crystal structure showing a possible configuration of the $\left[\left(\mathrm{SiO}_{4}\right)(\mathrm{OH})_{2}\right]$ cluster in the $P 2_{1} / a$ structure: when a Si1 site is occupied, the nearby equivalent position is vacant and two hydroxyls occur. (c) The serrated chains of Mnoctahedra, linked by Si-tetrahedra, in the $P 2_{1} / a$ structure model of leucophoenicite (mutually exclusive and symmetry-equivalent Si1 tetrahedra are shown in green) and $(d)$ the same structural view in the $P 2_{1}$ model, where the mutually exclusive and symmetry-independent Si11 and S12 tetrahedra are shown in light and dark green, respectively. These pictures have been created using Vesta software (Momma \& Izumi, 2011).

description of the high-pressure behavior of leucophoenicite may provide insights for the understanding of the elastic response and structural stability of the hydrous phase-B.

Leucophoenicite, ideally $\mathrm{Mn}_{7} \mathrm{Si}_{3} \mathrm{O}_{12}(\mathrm{OH})_{2}$, with minor, but frequent, substitutions of $\mathrm{Mn}$ by $\mathrm{Ca}$ and $\mathrm{Zn}$ (Moore, 1970; Dunn, 1985), has a structure built by the repetition along the $b$-axis of the hydrous double octahedral layers (Fig. 3). In the literature, leucophoenicite is always reported in the monoclinic $P 2_{1} / a$ space group, with $a \simeq 10.826 \AA, b \simeq 4.857 \AA, c \simeq$ $11.376 \AA, \beta \simeq 103.96^{\circ}$ and $V \simeq 580.4 \AA^{3}$ (Moore, 1970; White $\&$ Hyde, 1983a; Welch et al., 2002). With such a symmetry, one of the two independent tetrahedral sites (Si1) is only $1.8 \AA$ apart from an equivalent position, giving rise to a configuration with two edge-sharing $\mathrm{SiO}_{4}$ tetrahedra, which are necessarily mutually exclusive (Fig. 3). When a Si1 site is occupied, the equivalent nearby position is vacant and two hydroxyl group are present instead (Fig. 3).

In the following, we report an in situ high-pressure singlecrystal X-ray diffraction analysis of leucophoenicite, with the double aim to (i) provide a description of the elastic behavior of a mineral structurally related to dense hydrous $\mathrm{Mg}$-silicates and (ii) validate the suitability of the Xpress beamline (d)

experimental setup for equations-ofstate determination and fine-structural analysis.

A single-crystal fragment $(\sim 40 \mu \mathrm{m} \times$ $20 \mu \mathrm{m} \times 20 \mu \mathrm{m}$ ) of a natural sample of leucophoenicite from Franklyn (NJ, USA) has been selected for the experiment and loaded, along with two ruby spheres, in the pressure chamber $(250 \mu \mathrm{m}$ in diameter) obtained by spark-erosion in a stainless-steel gasket foil, previously pre-indented to $\sim 70 \mu \mathrm{m}$. The high-pressure experiment was conducted using a membranedriven Almax DAC, mounting BoehlerAlmax-type diamonds with $600 \mu \mathrm{m}$ culet diameter. The General Electric PACE 5000 pressure controller, available at the beamline, was used to remotely control the pressure increase and decrease exerted on the membrane. A mixture of methanol and ethanol (4:1), with a hydrostatic limit of $9.8 \mathrm{GPa}$ (Angel et al., 2007), was adopted as pressure-transmitting medium and loaded in the pressure chamber using a syringe. Pressure has been measured by the ruby-fluorescence method (Mao et al., 1986; Chervin et al., 2001), by using the Betsa PRL spectrometer available at the beamline.

Seven data collection runs were performed, at 0.16, 0.46, 1.14, 2.09, 2.68, 4.13 and $5.37 \mathrm{GPa}$, using a monochromatic $(\lambda=0.4957 \AA)$ incident beam, $80 \mu \mathrm{m}$ in diameter. The same collection strategy has been always adopted: 56 frames were collected by rotating the DAC around the $\omega$-axis from $-28^{\circ}$ to $+28^{\circ}$, with $1^{\circ}$ per frame step and $4 \mathrm{~s}$ per frame as exposure time. The diffraction patterns were collected by the MAR345 image plate detector at $214 \mathrm{~mm}$ from the sample position and operating with full-area mode and $150 \mu \mathrm{m} \times 150 \mu \mathrm{m}$ pixel size (mar2300 file format). The experimental data were analyzed using the Crysalis software (Rigaku Oxford Diffraction, 2018) for indexing, unit-cell refinement and peaks intensity reduction.

The refined unit-cell parameters, along with their uncertainties, are reported in Table 3, whereas the $V-P$ pattern, shown in Fig. 4(a), has been used for the equation-of-state determination by means of the EoSFit7_GUI software (Gonzalez-Platas et al., 2016). The Birch-Murnaghan equation of state (BM-EoS; Birch, 1947), which is based on the assumption that the strain energy of a solid undergoing compression can be expressed as a Taylor series in the Eulerian finite strain, has been adopted to describe the compressional behavior of leucophoenicite. More details on the BM-EoS and on its use in describing mineral elastic behavior can be found in Angel (2000). The quasi-horizontal 
Table 3

Lattice parameters of leucophoenicite at different pressures.

Standard deviations are given in parentheses.

\begin{tabular}{llllll}
\hline$P(\mathrm{GPa})$ & $a(\AA)$ & $b(\AA)$ & $c(\AA)$ & $\beta\left({ }^{\circ}\right)$ & $V\left(\AA^{3}\right)$ \\
\hline $0.16(2)$ & $10.883(2)$ & $4.871(2)$ & $11.419(2)$ & $103.90(2)$ & $587.7(4)$ \\
$0.46(2)$ & $10.875(2)$ & $4.865(2)$ & $11.4111(9)$ & $103.89(2)$ & $586.1(3)$ \\
$1.14(2)$ & $10.834(4)$ & $4.865(3)$ & $11.391(2)$ & $103.84(3)$ & $582.9(5)$ \\
$2.09(2)$ & $10.801(2)$ & $4.849(2)$ & $11.360(2)$ & $103.88(2)$ & $577.7(4)$ \\
$2.68(2)$ & $10.782(2)$ & $4.842(2)$ & $11.344(2)$ & $103.86(2)$ & $575.0(3)$ \\
$4.13(2)$ & $10.721(2)$ & $4.830(2)$ & $11.301(2)$ & $103.87(2)$ & $568.2(3)$ \\
$5.37(2)$ & $10.683(3)$ & $4.828(3)$ & $11.270(2)$ & $103.79(2)$ & $564(1)$ \\
\hline
\end{tabular}

trend of the normalized pressure $\left[F_{\mathrm{e}}=P / 3 f_{\mathrm{e}}\left(1+2 f_{\mathrm{e}}\right)^{5 / 2}\right]$ versus the Eulerian finite strain $\left[f_{\mathrm{e}}=\left(V_{0} / V\right)^{2 / 3}-1\right]$ pattern $\left(F_{\mathrm{e}}-f_{\mathrm{e}}\right.$ plot), shown in Fig. 4(b), suggests that a second-order truncation of the BM-EoS is sufficient to describe the $V-P$ relationship, at ambient $T$, in leucophoenicite; as the calculated uncertainty of $F_{\mathrm{e}}$ intrinsically decreases with increasing pressure (at constant absolute measured uncertainties in $P$ and $V$ ), the data plotted in Fig. 4(b) are characterized by significant values of $\sigma\left(F_{\mathrm{e}}\right)$ in the low $f_{\mathrm{e}}$ range [corresponding to low pressures; see Angel (2000) for further details about the $F_{\mathrm{e}}-f_{\mathrm{e}}$
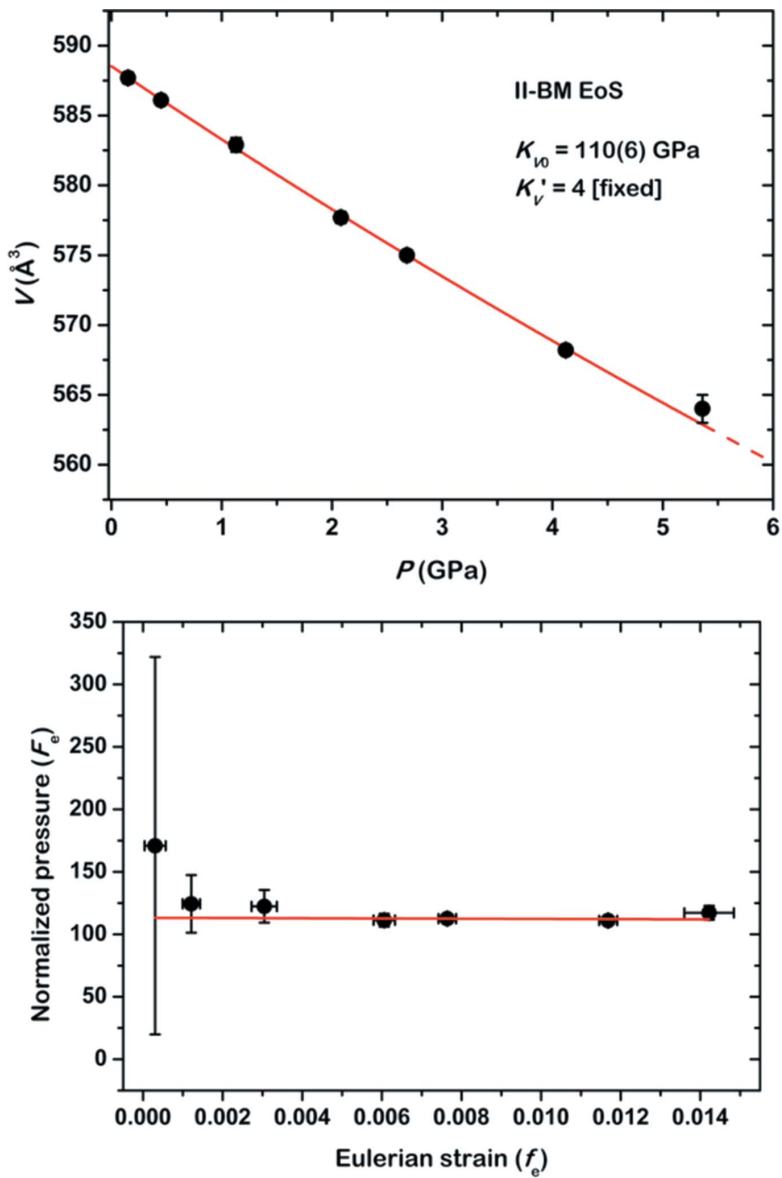

Figure 4

(Top) The high-pressure evolution of the unit-cell volume of leucophoenicite. The refined Birch-Murnaghan equation of state (truncated to the second order) is shown. (Bottom) The normalized pressure $\left(F_{\mathrm{e}}\right)$ versus the Eulerian strain $\left(f_{\mathrm{e}}\right)$ plot [see Angel (2000) for further details]. The quasi-horizontal linear fit suggests a second-order truncation of the BM-EoS. plot]. The fit to the experimental data yielded the following refined parameters: $V_{0}=588.5(7) \AA^{3}$ and $K_{V 0}=110(6) \mathrm{GPa}$, where $K_{V 0}=\beta_{V 0}^{-1}$ and $\beta_{V 0}=-(1 / V)(\partial V / \partial P)_{T}=$ 0.0090 (5) $\mathrm{GPa}^{-1}$. The refined II-BM EoS is shown in Fig. 4(a).

A preliminary single-crystal $\mathrm{X}$-ray diffraction investigation of a larger sample $(\sim 400 \mu \mathrm{m} \times 300 \mu \mathrm{m} \times 200 \mu \mathrm{m}$ in size $)$ was previously performed using an Xcalibur Oxford Diffraction diffractomer (at the Earth Sciences Department of the University of Milano, Italy), equipped with a conventional Mo X-ray tube and a CCD detector. The collected diffraction pattern could be satisfactorily described adopting the $P 2_{1} / a$ space group reported in the literature for leucophoenicite (Moore, 1970; Welch et al., 2002), as, of the 262 experimentally collected $h 0 l$ reflections with $h=2 n+1$, only 10 violated the extinction condition [i.e. with measured intensity $>3 \sigma(I)]$. The diffraction pattern of the significantly smaller crystal collected at the Xpress beamline using high-brilliance synchrotron radiation suggests that the actual symmetry of the investigated sample is $P 2_{1}$, as, of the 83 experimentally collected $h 0 l$ reflections with $h=2 n+1,54$ violated the extinction condition (Fig. 5). Starting from the coordinates reported by Moore (1970), the $P 2_{1}$ structure model of leucophoenicite was derived using the tools implemented in the JANA2006 software (Petrǐček et al., 2014). In the $P 2_{1}$ model, the mutually exclusive and symmetry-equivalent Si1 atoms, $\sim 1.8 \AA$ apart, are split into two independent atoms, Si11 and Si12 (Fig. 3). The structure refinement based on the intensity data collected at $0.16 \mathrm{GPa}$ was performed using the JANA2006 software, adopting the scattering factors of neutral $\mathrm{Mn}, \mathrm{Si}$ and $\mathrm{O}$ for the ' $\mathrm{Mn}$ ', 'Si' and 'O' sites, respectively. In order to accomplish the increased number of variables due to the $P 2_{1} / a$-to- $P 2_{1}$ symmetry reduction and the limited available reciprocal lattice for the shadowing of the DAC metallic components [see Miletich et al. (2000) for further details], few restraints have been applied to the structure refinement. Namely, based on the refined bond distances at ambient conditions, the (Si21, Si22)-O distances have been restrained to $1.65 \pm 0.04 \AA$ and the (Si11, Si12)-O distances to $1.75 \pm 0.04$ and $1.55 \pm$ $0.04 \AA$ for the basal and apical oxygens, respectively [see Moore (1970) and Welch et al. (2002) for further details]; the Si11 and Si12 occupancies have been refined constraining their sum to 1.00; the $\mathrm{Mn}, \mathrm{Si}$ and $\mathrm{O}$ sites have been restrained to share the same respective isotropic displacement parameters. The refinement converged to an $R$ factor of $7.84 \%$ for 467 independent reflections (out of a total of 586) with $I / \sigma(I)>3$, without any significant correlation among the 80 refined parameters and residual peaks in the difference-Fourier synthesis of electron density. The refined occupancies of the Si11 and Si12 sites were 0.28 (3) and 0.72 (3), respectively, thus validating the deviation from the $P 2_{1} / a$ symmetry. In order to check the reliability of the refined occupancy parameters, structure refinements based on the experimental data collected at 0.46, 2.09 and $4.13 \mathrm{GPa}$ have been performed adopting the same restrictions previously described. After convergence, the Si11 and Si12 refined occupancies were constant within $1.5 \sigma$. The refined structure models (cif files) are deposited as supporting information. 

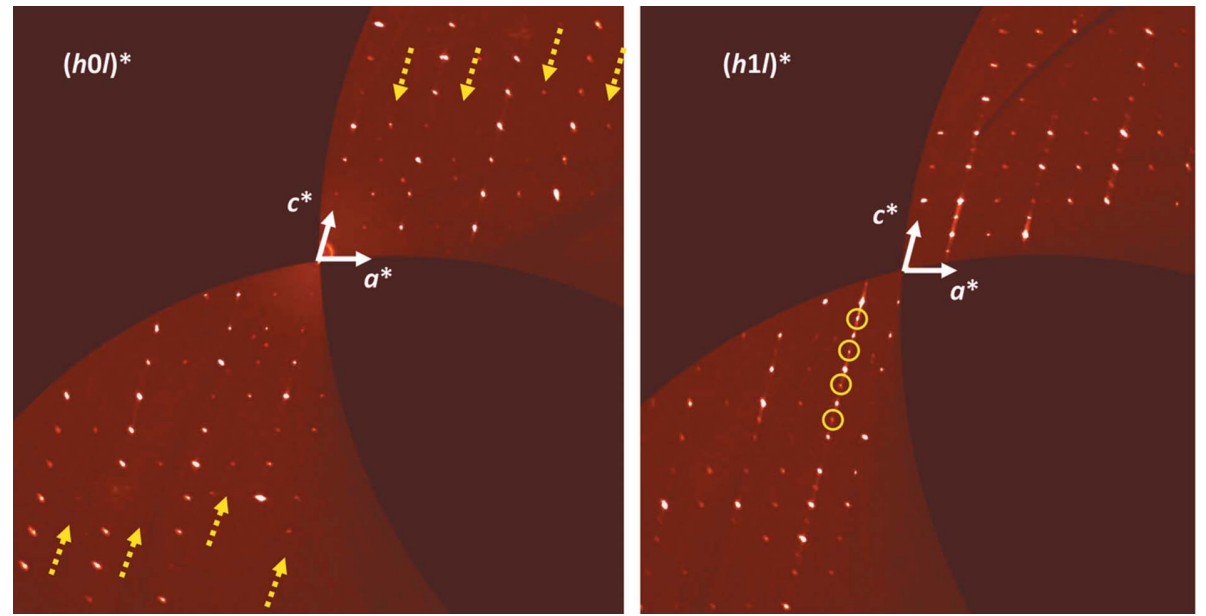

Figure 5

(Left) Reconstruction of the $(h 0 l) *$ reciprocal lattice plane, based on the leucophoenicite diffraction data collected at the Xpress beamline at 0.16 GPa. The dashed arrows point to rows with $h=2 n+1$, expected to be systematically absent for the $P 2_{1} / a$ space group. (Right) Reconstruction of the ( $h 1 l$ )* reciprocal lattice plane, based on the same experimental diffraction data previously described, showing the extra-peaks due to the (001) twinning (for an ease of clarity only few extra-peaks have been encircled) and the streaked reflections along the $\mathbf{c}^{*}$ axis.

A further feature, shared by all the diffraction patterns of the small leucophoenicite sample collected at the Xpress beamline but absent in the pattern collected using a conventional diffractometer on a larger crystal, is the occurrence of streaked reflections parallel to the $c^{*}$ reciprocal axis and additional peaks along the same direction. The latters are due to the occurrence of a (001) twinning already reported by Moore (1970) and White \& Hyde (1983b), whereas the formers can be attributed to the occurrence of faults along the serrated octahedra chains (White \& Hyde, 1983b).

\section{Conclusions}

The results reported in this study, based on in situ singlecrystal synchrotron X-ray diffraction data of clinoenstatite, $\mathrm{CaCO}_{3}$ polymorphs and leucophoenicite, validate the suitability of the Xpress beamline experimental setup for highpressure single-crystal XRD experiments. The quality of the experimental data allows: (i) to locate and characterize pressure-induced phase transitions, (ii) to solve ab initio the crystal structure of high-pressure polymorphs, even following a firstorder phase transition inducing several single-crystal domains (e.g. $\mathrm{CaCO}_{3}$-III), (iii) to perform fine structural analyses at the atomic scale, and (iv) to exploit the brilliance of a synchrotron $\mathrm{X}$-ray beam to disclose complex symmetry and structural features undetected when using conventional X-ray sources and larger crystals (e.g. leucophoenicite).

\section{Acknowledgements}

Alex Kurnosov (Bayerisches GeoInstitut) is thanked for the assistance in the neon-loading of the clinoenstatite DAC. Andrea Risplendente and Patrizia Fumagalli (Earth Sciences Department - University Milano) are thanked for the assistance in the synthesis of clinoenstatite. Luca Ziberna (University Trieste) and Juliette Maurice (University Milano) are thanked for the assistance during XRD experiments at
Xpress. Elettra Sincrotrone Trieste is acknowledged for the allocation of beam time through the long-term project 20155210 and for the provision of synchrotron facilities and travel support.

\section{Funding information}

PL, SM and MM acknowledge the support of the Italian Ministry of Education (MIUR) through the project 'Dipartimenti di Eccellenza 2018-2022' and of the University of Milano through the project 'Piano di Sostegno alla Ricerca 2018'. BJ thanks IISc Bangalore and ICTP Trieste for the award of IISc-ICTP fellowship.

\section{References}

Alabarse, F. G., Joseph, B., Lausi, A. \& Haines, J. (2019). Molecules, 24, 2864-2611.

Angel, R. J. (2000). Rev. Mineral. Geochem. 41, 35-59.

Angel, R. J., Bujak, M., Zhao, J., Gatta, G. D. \& Jacobsen, S. D. (2007). J. Appl. Cryst. 40, 26-32.

Angel, R. J. \& Hugh-Jones, D. A. (1994). J. Geophys. Res. 99, $19777-$ 19783.

Bassett, W. A. (2009). High Press. Res. 29, 163-186.

Birch, F. (1947). Phys. Rev. 71, 809-824.

Boffa Ballaran, T., Kurnosov, A. \& Trots, D. (2013). High Press. Res. 33, 453-465.

Boldyreva, E. V. (2008). Acta Cryst. A64, 218-231.

Bridgman, P. W. (1939). Am. J. Sci. 237, 7-18.

Chervin, J. C., Canny, B. \& Mancinelli, M. (2001). High Press. Res. 21, 302-314.

Davis, B. L. (1964). Science, 145, 489-491.

Dera, P. (2010). High-Pressure Crystallography: From Fundamental Phenomena to Technological Applications, edited by E. Boldyreva \& P. Dera, pp. 11-22. Dordrecht: Springer Netherlands.

Dera, P., Zhuravlev, K., Prakapenka, V., Rivers, M. L., Finkelstein, G. J., Grubor-Urosevic, O., Clark, S. M. \& Downs, R. T. (2013). High Press. Res. 33, 466-484.

Dubrovinsky, L., Boffa-Ballaran, T., Glazyrin, K., Kurnosov, A., Frost, D. J., Merlini, M., Hanfland, M., Prakapenka, V. B., Schouwink, P., Pippinger, T. \& Dubrovinskaia, N. (2010). High Press. Res. 30, 620-633. 
Duffy, T. S. \& Wang, Y. (1998). Rev. Mineral. 33, 425-457.

Dunn, P. J. (1985). Am. Mineral. 70, 379-387.

Finger, L. W., Ko, J., Hazen, R. M., Gasparik, T., Hemley, R. J., Prewitt, C. T. \& Weidner, D. J. (1989). Nature, 341, 140-142.

Fiquet, G., Guyot, F. \& Itie, J.-P. (1994). Am. Mineral. 79, 15-23.

Gonzalez-Platas, J., Alvaro, M., Nestola, F. \& Angel, R. (2016). J. Appl. Cryst. 49, 1377-1382.

Hagiya, K., Matsui, M., Kimura, Y. \& Akahama, Y. (2005). J. Mineral. Petrol. Sci. 100, 31-36.

Hamilton, D. L. \& Henderson, C. M. B. (1968). Mineral. Mag. 36, 832838.

Lazarz, J. D., Dera, P., Hu, Y., Meng, Y., Bina, C. R. \& Jacobsen, S. D. (2019). Am. Mineral. In the press.

Liu, L.-G. \& Mernagh, T. P. (1990). Am. Mineral. 75, 801-806.

Makovicky, E. (1995). Am. Mineral. 80, 676-679.

Mao, H. K., Xu, J. \& Bell, P. M. (1986). J. Geophys. Res. 91, 46734676.

McMahon, M. I., Loa, I., Stinton, G. W. \& Lundegaard, L. F. (2013). High Press. Res. 33, 485-500.

McMillan, P. F. (2002). Nat. Mater. 1, 19-25.

Merlini, M. \& Hanfland, M. (2013). High Press. Res. 33, 511-522.

Merlini, M., Hanfland, M. \& Crichton, W. A. (2012). Earth Planet. Sci. Lett. 333-334, 265-271.

Merlini, M., Hanfland, M., Salamat, A., Petitgirard, S. \& Müller, H. (2015). Am. Mineral. 100, 2001-2004.
Merrill, L. \& Bassett, W. A. (1974). Rev. Sci. Instrum. 45, 290-294.

Merrill, L. \& Bassett, W. A. (1975). Acta Cryst. B31, 343-349.

Miletich, R., Allan, D. R. \& Kuhs, W. F. (2000). Rev. Mineral. Geochem. 41, 445-519.

Momma, K. \& Izumi, F. (2011). J. Appl. Cryst. 44, 1272-1276.

Moore, P. B. (1970). Am. Mineral. 55, 1146-1166.

Oganov, A. R., Glass, C. W. \& Ono, S. (2006). Earth Planet. Sci. Lett. 241, 95-103.

Oszlányi, G. \& Sütő, A. (2004). Acta Cryst. A60, 134-141.

Palatinus, L. \& Chapuis, G. (2007). J. Appl. Cryst. 40, 786-790.

Petř̌ček, V., Dušak, M. \& Palatinus, L. (2014). Z. Kristallogr. 229, 345-352.

Rigaku Oxford Diffraction (2018). CrysAlisPro, version 1.171.38.46. Rigaku Corporation, Oxford, UK.

Smyth, J. R. \& Ahrens, T. J. (1997). Geophys. Res. Lett. 24, 1595-1598.

Suito, K., Namba, J., Horikawa, T., Taniguchi, Y., Sakurai, N., Kobayashi, M., Onodera, A., Shimomura, O. \& Kikegawa, T. (2001). Am. Mineral. 86, 997-1002.

Van Valkenburg, A. (1965). Conference Internationale sur-les-Hautes Pressions, 2-6 August 1965, Le Creusot, Saone-et-Loire, France.

Welch, M. D., Marshall, W. G., Ross, N. L. \& Knight, K. S. (2002). Am. Mineral. 87, 154-159.

White, T. J. \& Hyde, B. G. (1983a). Am. Mineral. 68, 1009-1021.

White, T. J. \& Hyde, B. G. (1983b). Acta Cryst. B39, 10-17.

Yuan, H. \& Zhang, L. (2017). Matter Radiat. Extremes, 2, 117-128. 